\title{
Hypoxia-regulated neurotrophin-3 expression by multicopy hypoxia response elements reduces apoptosis in PC12 cells
}

\author{
JUNFENG ZHANG $^{1 *}$, QINDONG SHI $^{1,2^{*}}$, XINLIN CHEN $^{1}$, PENGBO YANG $^{1}$, CUNFANG $^{1}{ }^{1}$, JIANSHUI ZHANG $^{1}$, \\ HAIXIA LU ${ }^{1}$, JIANXIN LIU ${ }^{1}$, QIAN JIAO ${ }^{1}$, LINGYU ZHAO ${ }^{1}$, BINGQIAO ZHAO ${ }^{3}$, PING ZHENG ${ }^{3}$ and YONG LIU ${ }^{1}$ \\ ${ }^{1}$ Institute of Neurobiology, Environment and Genes Related to Diseases Key Laboratory of Education Ministry \\ and ${ }^{2}$ First Affiliated Hospital of Xi'an Jiaotong University College of Medicine, Xi'an, Shaanxi 710061; \\ ${ }^{3}$ The State Key Laboratory of Medical Neurobiology, Shanghai 200032, P.R. China
}

Received April 9, 2012; Accepted July 20, 2012

DOI: 10.3892/ijmm.2012.1119

\begin{abstract}
We have previously reported that 5 copies of the hypoxia response element (HRE) can conditionally regulate brain-derived neurotrophic factor gene expression under hypoxic/ischemic conditions in mice. In the present study, we investigated the controlled expression of neurotrophin-3 (NT-3) by HRE under hypoxic conditions and determined the protective effects of conditionally expressed NT-3 on hypoxia-induced apoptosis in PC12 cells. Five copies of the HRE (5HRE) and the simian virus 40 minimal promoter (SV40mp) were employed to construct a cassette, and transfer of therapeutic gene, NT-3, into PC12 cells was achieved using a retroviral vector. Our results showed that the retroviral vector, pLNC-5HRE-NT3, was successfully constructed and transfected into PC12 cells. Compared with normal conditions, in which NT-3 was expressed at low levels, the expression of NT-3 significantly increased under hypoxic conditions in 5HRE-NT3 transgenic PC12 cells $(\mathrm{P}<0.05)$. By contrast, in NT-3 transgenic PC12 cells without HRE, we found no significant difference in NT-3 expression between the normoxic and hypoxic groups. The conditional adjustment of NT-3 expression by $5 \mathrm{HRE}$ significantly reduced apoptosis induced by hypoxia in 5HRE-NT3 transgenic $\mathrm{PC} 12$ cells $(\mathrm{P}<0.05)$ but not in 5HRE-enhanced green fluorescent protein (EGFP) transgenic PC12 cells and PC12 cells without gene transfer. In addition, the hypoxia-induced upregulation of both $\mathrm{p} 38$ and caspase-3 activities was suppressed in 5HRE-NT3 transgenic $\mathrm{PC} 12$ cells under hypoxic conditions $(\mathrm{P}<0.05)$. Taken together, these results demonstrate that $5 \mathrm{HRE}-\mathrm{SV} 40 \mathrm{mp}$ regulates NT-3
\end{abstract}

Correspondence to: Professor Yong Liu, Institute of Neurobiology, Environment and Genes Related to Diseases Key Laboratory of Education Ministry, Xi'an Jiaotong University College of Medicine, 76 Yanta West Road, Xi'an, Shaanxi 710061, P.R. China

E-mail: liuy5599@163.com

${ }^{*}$ Contributed equally

Key words: hypoxia response element, neurotrophin-3, gene therapy, hypoxia, apoptosis gene expression in response to hypoxia in $\mathrm{PC} 12$ cells. The data presented in this study may prove useful in future gene therapy studies for the treatment of ischemic diseases.

\section{Introduction}

To achieve the optimal effects of gene therapy, it is crucial to develop a strategy to conditionally express the therapeutic gene of interest and reduce any unexpected side-effects resulting from excessive expression of the exogenous gene $(1,2)$. Previous studies have suggested that multiple copies of the hypoxia response element (HRE) can regulate the expression of therapeutic genes in response to hypoxia (3-6). The hypoxia inducible factor-1 (HIF-1) is a transcription factor that plays a key role in the regulation of gene expression under hypoxic conditions (7). HIF-1 is composed of 2 subunits, HIF-1 $\alpha$ and HIF-1 $\beta$. Under normoxic conditions, HIF-1 $\alpha$ is rapidly degraded by the ubiquitin-proteasome pathway. However, under hypoxic conditions, HIF- $1 \alpha$ becomes stabilized and forms an active HIF-1 heterodimer with HIF-1 $\beta$. Through its binding to the HRE located in the regulatory domain of target genes, HIF-1 $\alpha$ can upregulate the expression of several hypoxiarelated genes in response to hypoxia (8). Thus, HRE can regulate downstream gene expression according to the activity of HIF-1, which is affected by the oxygen concentration.

Neurotrophin-3 (NT-3) not only activates its own specific orphan receptor, TrkC, but also activates TrkA and TrkB to mediate almost all of its neuron survival and differentiationpromoting activities in the central and peripheral nervous system (9). In vitro, exogenous NT-3 promotes the proliferation and survival of neural stem cells and increases the percentage of neural stem cells that differentiate into neurons (10-12). In vivo, NT-3 facilitates angiogenesis and neurogenesis by which functional recovery can be promoted after stroke (13-15). These results suggest that NT-3 may be an optimal gene to promote neuroprotection against a number of brain injuries including cerebral ischemia.

In the present study, we generated a recombinant retroviral vector expressing NT-3 under the control of a cassette constructed by 5 copies of the HRE and the simian virus 40 minimal promoter (5HRE-SV40mp). The hypoxia-regulated NT-3 expression was confirmed, and the protective effects of 
hypoxia-regulated NT-3 against apoptosis were demonstrated in PC12 cells. Moreover, the activities of p38 and caspase-3, which may be involved in these effects, were investigated.

\section{Materials and methods}

Cell culture and hypoxia treatment. PT67 cells (American Type Culture Collection) were cultured in DMEM (Gibco) supplemented with $10 \%$ fetal bovine serum (Gibco), $100 \mathrm{U} / \mathrm{ml}$ penicillin and $100 \mathrm{U} / \mathrm{ml}$ streptomycin (Sigma) at $37^{\circ} \mathrm{C}$ in a humidified incubator containing 95\% air and $5 \% \mathrm{CO}_{2} . \mathrm{PC} 12$ cells were maintained in DMEM supplemented with $10 \%$ horse serum (HyClone), 5\% fetal bovine serum, $100 \mathrm{U} / \mathrm{ml}$ penicillin and $100 \mathrm{U} / \mathrm{ml}$ streptomycin. For immunocytochemistry and in situ cell death detection, the cells were plated onto poly-L-lysine-coated coverslips.

On day 2 after plating, the cells were placed in an anaerobic workstation (Bugbox; Ruskinn Technology) and perfused with a gas mixture of $5 \% \mathrm{CO}_{2}, 0.3 \% \mathrm{O}_{2}$ and $94.7 \% \mathrm{~N}_{2}$ to induce hypoxia. For HIF-1 $\alpha$ detection, the cells were exposed to hypoxia for 3, 6, 12, 24 and $48 \mathrm{~h}$. For hypoxia-regulated NT-3 expression experiments and the apoptosis assay, the cells were exposed to hypoxia for $48 \mathrm{~h}$.

Construction of recombinant retroviral vector. The full length coding sequences of human NT-3, 5 copies of the vascular endothelial growth factor (VEGF) HRE consensus sequence (CCACAGTGCATACGTGGGCTCCAACAGGTCCTCTT), SV40mp and enhanced green fluorescent protein (EGFP) were cloned into the retroviral vector, pLNCX (Clontech), between 2 long terminal repeats to generate pLNC-5HRE-SV40-EGFP, pLNC-SV40-NT3 and pLNC-5HRE-SV40-NT3 (Fig. 1A).

Generation of transgenic PC12 cells. To generate retroviruses that can mediate 5HRE-EGFP, NT-3 or 5HRE-NT3 gene transfer, the PT67 packaging cells were transfected with the constructed plasmids using Lipofectamine 2000 (Invitrogen). After 2 days, the virus-containing supernatants were filtered through a $0.45-\mu \mathrm{m}$ cellulose acetate filter and added to PC12 cells (American Type Culture Collection) for $24 \mathrm{~h}$. The medium was then replaced with DMEM containing $10 \%$ horse serum and 5\% fetal bovine serum. After 24 h, G418 (Invitrogen) was added to the medium to screen and select for individual colonies. The colonies were identified using reverse transcription PCR (RT-PCR), immunocytochemistry and western blot analysis. The transgenic PC12 cells were designated as PC12-5HRE-EGFP, PC12-NT3 and PC125HRE-NT3.

$R T$-PCR. Total RNA was isolated from cells using the TRIzol LS reagent (Invitrogen), and first-strand cDNA was synthetized using the PrimeScript ${ }^{\mathrm{TM}}$ RT reagent kit (Takara Bio, Inc.). PCR reactions were performed using the Premix Taq Version 2.0 (Takara Bio, Inc.). To detect the exogenous genes, $\beta$-actin mRNA was used as the internal control and the following primers were used: $\beta$-actin, 5'-GGAGATTACTGCCCTGG CTCCTA-3' (forward) and 5'-GACTCATCGTACTCCTGC TTGCTG-3' (reverse); SV40mp, 5'-CGGGATCCGTTAACT CTGCGATCTGC-3' (forward) and 5'-CGGTGAAGATCTCT GCAGAATTCGAAGC-3' (reverse); NT-3, 5'-GGCAGATCT
GGTGATGTCCATCTTG-3' (forward) and 5'-GCCGAGC TCTCATGTTCTTCCGATT-3' (reverse); and EGFP, 5'-CGG GATCCAGATCTCGCCACCATG-3' (forward) and 5'-CCA TCGATGGTTACTTGTACAGCTCGTCC-3' (reverse). The reactions were performed in a total volume of $20 \mu \mathrm{l}$ with an initial cycle at $94^{\circ} \mathrm{C}$ for $2 \mathrm{~min}$, followed by 30 cycles as follows: $94^{\circ} \mathrm{C}$ for $30 \mathrm{sec}, 60^{\circ} \mathrm{C}$ for $30 \mathrm{sec}$ and $72^{\circ} \mathrm{C}$ for $1 \mathrm{~min}$. The amplified PCR products were separated on $1.5 \%$ agarose gels and subsequently imaged. For a semiquantitative analysis of NT-3 mRNA, the band intensities were measured using the Image J software (version $1.43 \mathrm{u}$, National Institutes of Health), and the data were normalized to the level of $\beta$-actin in the same sample.

Immunocytochemistry. The cells were fixed with $4 \%$ paraformaldehyde for $20 \mathrm{~min}$, blocked with $5 \%$ normal goat serum for $1 \mathrm{~h}$ and labeled with rabbit monoclonal anti-NT-3 primary antibody (1:100; Santa Cruz Biotechnology, Inc.) and $\mathrm{Cy} 3$-conjugated goat anti-rabbit IgG secondary antibody (1:500; Vector Laboratories). After counterstaining with DAPI (Sigma), the cells were imaged using a fluorescence microscope (Olympus BX51).

Western blot analysis. The cells were harvested and lysed in RIPA lysis buffer (Pierce Biotechnology, Inc.) supplemented with a protease inhibitor cocktail (Roche Diagnostics). The lysate was centrifuged at $12,000 \mathrm{rpm}$ for $10 \mathrm{~min}$ at $4^{\circ} \mathrm{C}$, and the protein concentration of the supernatant was determined using a BCA protein assay kit (Pierce Biotechnology, Inc.). Equal amounts of protein from each lysate were separated using 12\% SDS-PAGE, and transferred onto a nitrocellulose membrane. The membrane was blocked with $5 \%$ non-fat milk in TBS-T (50 mM Tris, $150 \mathrm{mM} \mathrm{NaCl}, \mathrm{pH}$ 7.6, 0.1\% Tween-20) for $4 \mathrm{~h}$. The following primary antibodies were incubated with the membrane overnight at $4^{\circ} \mathrm{C}$ : mouse monoclonal antiHIF-1 $\alpha$ (1:2000; Abcam), rabbit monoclonal anti-NT-3 (1:200, Santa Cruz Biotechnology, Inc.), rabbit polyclonal anti-active caspase-3 (1:1000; Abcam), rabbit monoclonal anti-P-p38 (1:1000; Cell Signaling Technology, Inc.), rabbit monoclonal anti-p38 (1:2000; Cell Signaling Technology, Inc.) and mouse monoclonal anti- $\beta$-actin (1:5000; Santa Cruz Biotechnology, Inc.). The immunoreactive bands were visualized by enhanced chemiluminescence (Pierce Biotechnology, Inc.) using a horseradish peroxidase-labeled secondary anti-rabbit/mouse antibody (1:2000; Santa Cruz Biotechnology, Inc.).

Enzyme-linked immunosorbent assay (ELISA). The cells were incubated for $48 \mathrm{~h}$ under normoxic or hypoxic conditions. The NT-3 levels in the PC12-NT3 and PC12-5HRE-NT3conditioned medium were determined using an ELISA kit for human NT-3 (Promega) according to the manufacturer's instructions.

In situ cell death detection. Terminal dUTP nick end-labeling (TUNEL) assay was performed to determine apoptosis using an In situ Cell Death Detection kit (Roche Diagnostics). The cells were fixed with $4 \%$ paraformaldehyde for $1 \mathrm{~h}$, permeabilized with $0.1 \%$ Triton $\mathrm{X}-100$ in $0.1 \%$ sodium citrate for 2 min on ice, incubated with $50 \mu 1$ TUNEL reaction mixtures for $1 \mathrm{~h}$ at $37^{\circ} \mathrm{C}$ and counterstained with DAPI (Sigma) for 
A

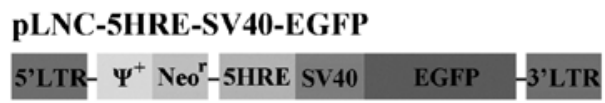

pLNC-SV40-NT3

5'LTR- $\Psi^{+}$Neo ${ }^{r}-$ SV40 NT3 IRES EGFP - 3'LTR

pLNC-5HRE-SV40-NT3

5'LTR- $\Psi^{+}$Neo $^{\mathrm{r}}-5$ HRE SV40 NT3 IRES EGFP -3'LTR

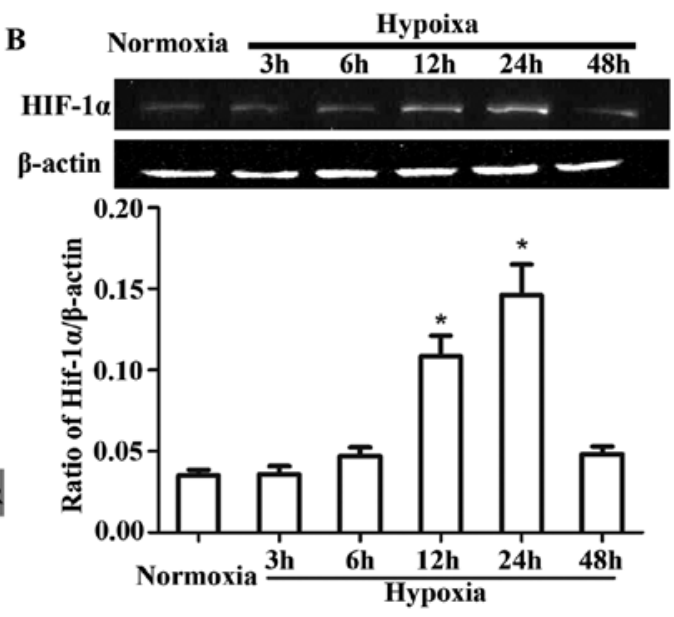

Figure 1. Schematic diagrams (A) show the structure of the recombinant retroviral vectors. LTR, Long terminal repeat; $\psi^{+}$, Extended packaging signal; Neo ${ }^{\mathrm{r}}$, Neomycin resistance gene; IRES, Internal ribozyme entry site. Image (B) shows the western blot analysis of HIF-1 $\alpha$ expression after hypoxia at $3,6,12,24$ and $48 \mathrm{~h}$ or normoxia in PC12 cells. HIF-1 $\alpha$ expression increased significantly following hypoxia at 12 and $24 \mathrm{~h}$. $\mathrm{n}=3$. ${ }^{*} \mathrm{P}<0.05$ vs. normoxic group.

5 min. Three microscopic fields (x20 magnification) of TUNEL-positive cells on 3 separate coverslips were selected and imaged. The number of TUNEL-positive cells was then counted and normalized to the total number of cells in the images captured from these areas. The percentage of positive cells was calculated as the mean of the percentages obtained from 9 images.

Statistical analysis. The data are presented as the means \pm SD, and the statistical analysis was performed using ANOVA followed by Fisher's least significant difference test. A probability value of $<0.05$ was considered statistically significant.

\section{Results}

Detection of HIF-1 $\alpha$ expression after hypoxic treatment in PC12 cells. Western blot analyses showed that HIF-1 $\alpha$ was expressed at low levels under normoxic conditions and increased after hypoxic treatment in PC12 cells (Fig. 1B). Although HIF-1 $\alpha$ expression had not significantly increased after hypoxic treatment in the 3- or 6-h groups, compared with the normoxic group, it did significantly increase after hypoxia in the 12- and 24-h groups $(\mathrm{P}<0.05)$, and peaked at $24 \mathrm{~h}$. These data demonstrate the feasibility of using HRE to regulate NT-3 expression in response to hypoxia.

Identification of transgenic PC12 cells. In this study, we successfully constructed recombinant retroviral vectors and generated transgenic PC12 cells. By combining fluorescence with light microphotographs (Fig. 2A), we revealed that all of the transgenic cells were EGFP-positive, whereas no EGFP was detected in the PC12 cells without gene transfer. The RT-PCR results (Fig. 2B) showed mRNA expression of SV40mp and EGFP in all of the transgenic cells, and NT-3 mRNA expression was detected in the PC12-NT3 and PC125HRE-NT3 cells but not in the PC12-5HRE-EGFP or PC12 cells without gene transfer. Immunocytochemistry demonstrated that NT-3 and EGFP were co-labeled in PC12-NT3 and PC12-5HRE-NT3 cells, but no NT-3-positive staining could be detected in PC12-5HRE-EGFP or PC12 cells without gene transfer (Fig. 2C), which was consistent with our RT-PCR results.

Verification of hypoxia-regulated NT-3 expression in PC12$5 H R E-N T 3$ cells. To determine whether 5 copies of HRE can conditionally control NT-3 gene expression, we performed RT-PCR, western blot analysis and an ELISA assay to examine the mRNA and protein expression of NT-3. Under normoxic conditions, a very low level of NT-3 mRNA expression was observed in PC12-5HRE-NT3 cells, and this level was significantly lower than that in the $\mathrm{PC} 12-\mathrm{NT} 3$ group $(\mathrm{P}<0.05)$ (Fig. 3A). However, NT-3 mRNA expression was significantly increased in PC12-5HRE-NT3 cells after hypoxic treatment compared with the normoxic group $(\mathrm{P}<0.05)$. By contrast, NT-3 mRNA expression was decreased in PC12-NT3 cells after hypoxic treatment compared with the normoxic group $(\mathrm{P}<0.05)$. The western blot analysis results (Fig. 3B) revealed that the NT-3 protein was also expressed at an extremely low level in PC12-5HRE-NT3 cells, which was significantly lower than that in the PC12-NT3 group under normoxic conditions $(\mathrm{P}<0.05)$. After hypoxic treatment, NT-3 protein expression was significantly increased in PC12-5HRE-NT3 cells compared with the normoxic group $(\mathrm{P}<0.05)$. We found no significant difference in the level of NT-3 protein expression between the normoxic and hypoxic group in PC12-NT3 cells.

As a secreted protein, NT-3 can be detected in conditional medium with the use of an ELISA assay (Fig. 3C). Our results showed that the NT-3 protein in PC12-5HRE-NT3 cells was detected at $315.79 \pm 10.94 \mathrm{pg} / \mathrm{ml}$, which was significantly lower than the level in PC12-NT3 cells $(3702.28 \pm 163.48, \mathrm{P}<0.05)$ under normoxic conditions. Following hypoxic treatment, NT-3 protein expression significantly increased to a high level of $3361.26 \pm 77.86 \mathrm{pg} / \mathrm{ml}$, which was approximately 10 -fold greater than that in the normoxic group $(\mathrm{P}<0.05)$. Taken together, these data suggest that NT-3 expression was effectively increased by 5HRE in PC12-5HRE-NT3 cells in response to hypoxia.

Reduced apoptosis in 5HRE-NT3 transgenic PC12 cells after hypoxia. Although only a few cells were TUNEL-positive 


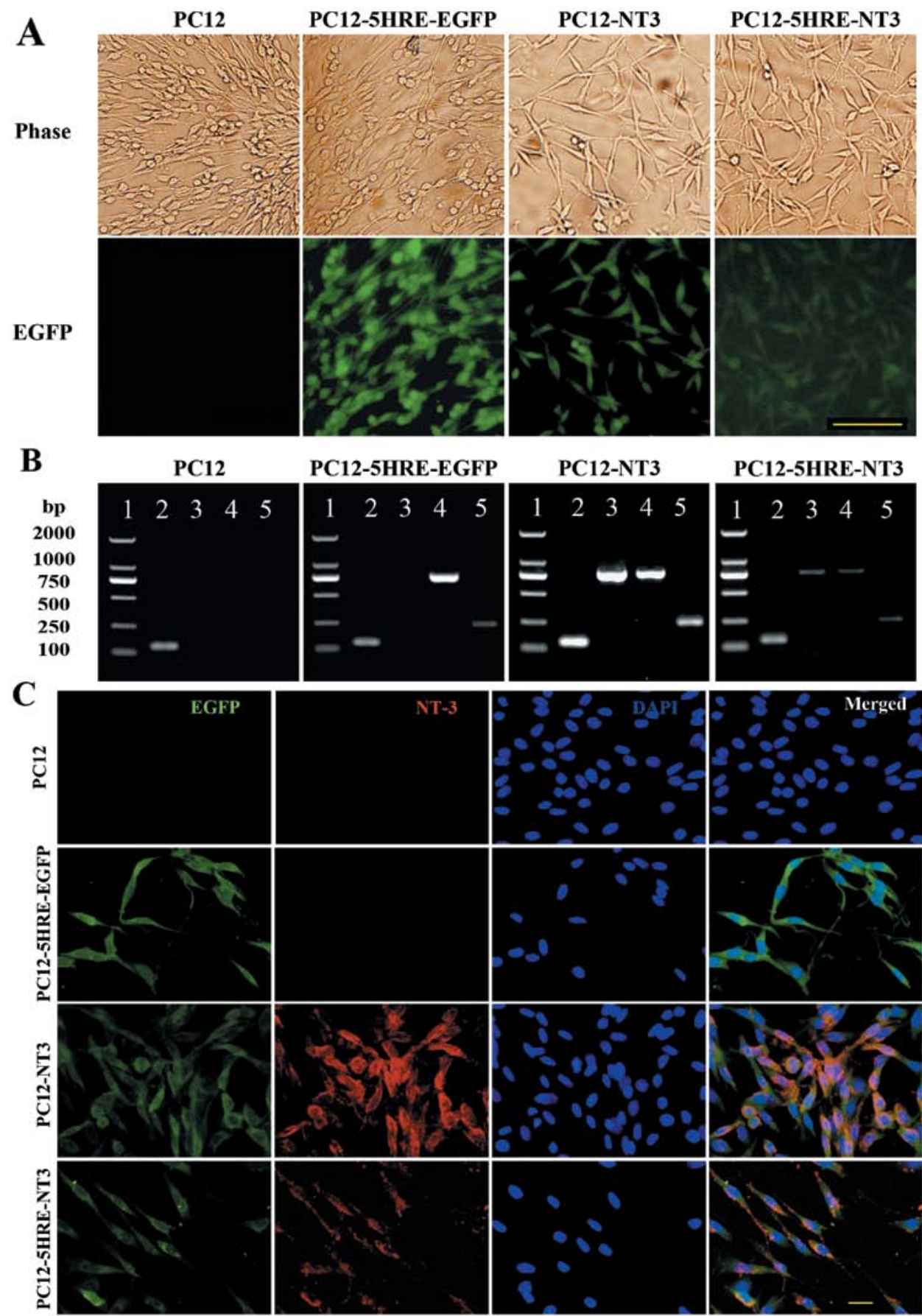

Figure 2. (A) Identification of transgenic PC12 cells. EGFP fluorescence was observed in PC12-5HRE-EGFP, PC12-NT3 and PC12-5HRE-NT3 cells. Green shows EGFP; scale bar, $100 \mu \mathrm{m}$. (B) Simultaneously, the expression of the exogenous genes in the PC12 and transgenic PC12 cells was detected by RT-PCR. Lane 1, DNA molecular weight marker; lane $2, \beta$-actin PCR product; lane 3, NT-3 PCR product; lane 4, EGFP PCR product; lane 5, SV40mp PCR product. (C) Immunocytochemistry with an antibody against NT-3. NT-3 (red) was detected in PC12-NT3 and PC12-5HRE-NT3 cells. Green shows EGFP; blue shows DAPI; merged, EGFP/NT-3/DAPI. Scale bar, $20 \mu \mathrm{m}$.

under normoxic conditions (Fig. 4A and B), the percentage of TUNEL-positive cells had significantly increased after $48 \mathrm{~h}$ of hypoxic treatment in the $\mathrm{PC} 12$ cell group $(\mathrm{P}<0.05)$. However, the percentage of TUNEL-positive cells in the PC12-5HRE-NT3 group was significantly reduced compared to the $\mathrm{PC} 12$ cell group under hypoxic conditions $(\mathrm{P}<0.05)$, whereas the percentage of TUNEL-positive cells was not significantly different between the PC12-5HRE-EGFP and PC12 groups under hypoxic conditions.
Suppressed activation of $p 38$ and caspase- 3 is accompanied with a decrease in PC12 cell apoptosis. The phosphorylation of p38 and activation of caspase- 3 were detected by western blot analysis to determine whether these proteins were involved in the reduction of apoptosis mediated by gene transfer in PC12 cells. Compared with the PC12 cells in the normoxic group, the phosphorylation of $\mathrm{p} 38(\mathrm{P}<0.05)$ (Fig. 4C) and activation of caspase-3 $(\mathrm{P}<0.05)$ (Fig. 4D) were significantly increased in PC12 cells after $48 \mathrm{~h}$ of hypoxia. However, the phosphory- 
A

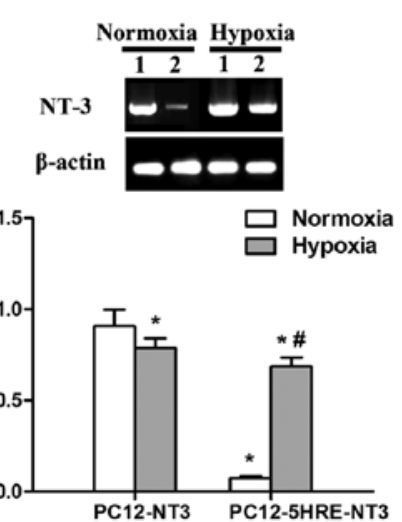

B

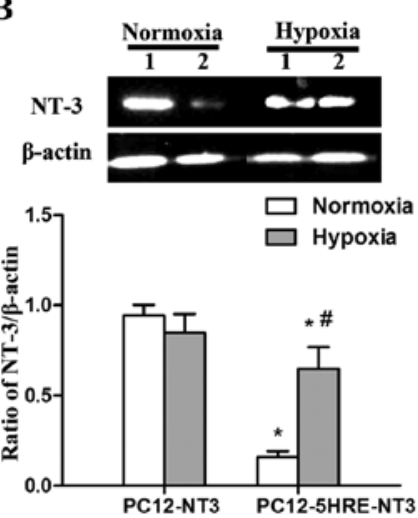

C

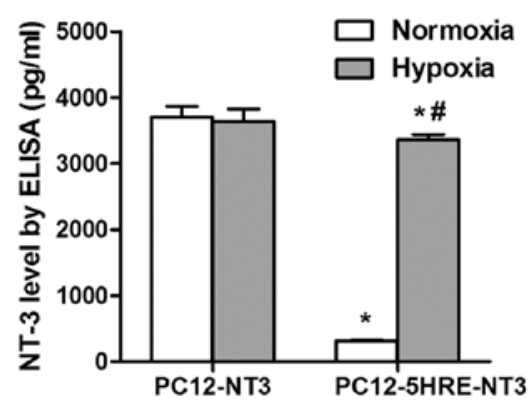

Figure 3. Expression of NT-3 in PC12-NT3 and PC12-5HRE-NT3 cells. (A) RT-PCR and (B) western blot analyses show the levels of NT-3 mRNA and protein expressed in PC12-NT3 (lane 1) or PC12-5HRE-NT3 (lane 2) cells under normoxic or hypoxic conditions. (C) Bar graph shows the ELISA result for the level of NT-3 in the conditioned medium of the PC12-NT3 and PC12-5HRE-NT3 cells. $n=3 .{ }^{~} \mathrm{P}<0.05$ vs. PC12-NT3 normoxic group; ${ }^{~} \mathrm{P}<0.05$ vs. PC125HRE-NT3 normoxic group.

A

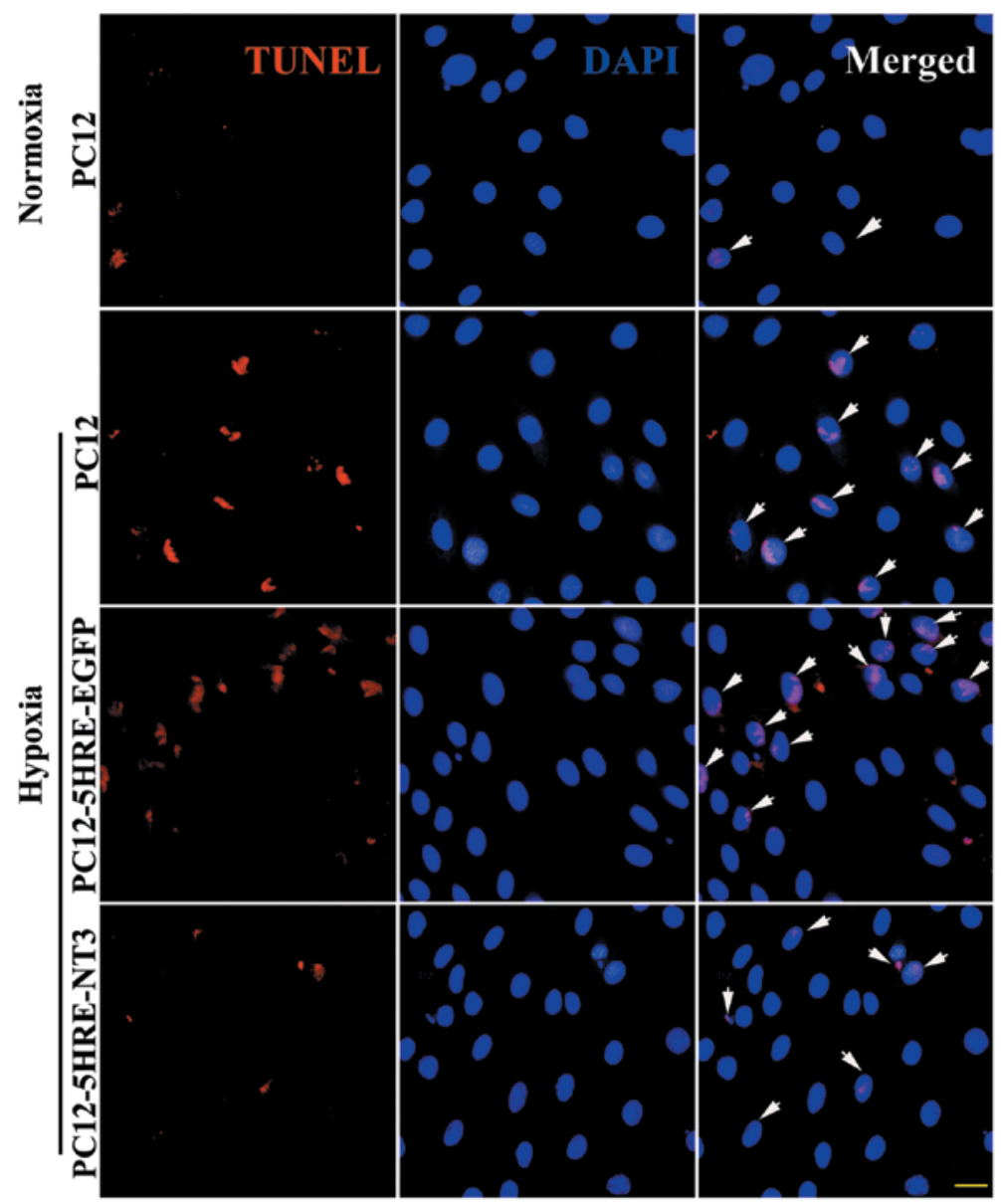

B
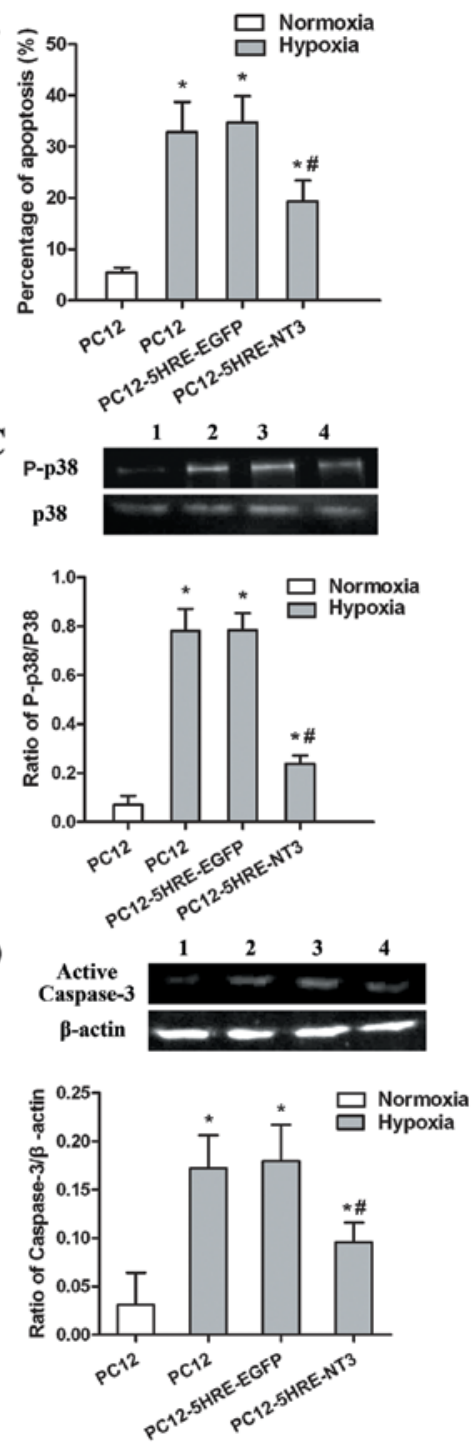

Figure 4. Decrease in apoptosis in PC12-5HRE-NT3 cells with reduced activation of p38 and caspase-3. (A) Red shows TUNEL staining revealed apoptosis in PC12 cells under normoxic conditions or in PC12, PC12-5HRE-EGFP and PC12-5HRE-NT3 cells after hypoxic injury for 48 h. Blue shows DAPI counterstaining; the arrows indicate TUNEL-positive cells. Scale bar, $20 \mu \mathrm{m}$. (B) Bar graph shows the statistical analysis of the TUNEL staining experiments. $\mathrm{n}=3$. ${ }^{*} \mathrm{P}<0.05$ vs. PC12 normoxic group; ${ }^{\text {P }}<0.05$ vs. PC12 hypoxic group. Western blot analysis shows a decreased activation of (C) p38 and (D) caspase-3 in PC12-5HRE-NT3 cells after hypoxic injury for $48 \mathrm{~h}$. Lane 1, PC12 normoxic group; lane 2, PC12 hypoxic group; lane 3, PC12-5HRE-EGFP hypoxic group; lane 4, PC12-5HRE-NT3 hypoxic group. ${ }^{*} \mathrm{P}<0.05$ vs. PC12 normoxic group; ${ }^{*} \mathrm{P}<0.05$ vs. $\mathrm{PC} 12$ hypoxic group. 
lation of p38 and activation of caspase-3 were remarkably decreased in the PC12-5HRE-NT3 group compared with the PC12 cells under hypoxic conditions $(\mathrm{P}<0.05)$. There was no significant difference in the phosphorylation of p38 and the activation of caspase-3 between the PC12-5HRE-EGFP and $\mathrm{PC} 12$ cell groups under hypoxic conditions. These data indicate that both $\mathrm{p} 38$ and caspase- 3 may participate in the protective effects of 5HRE-NT3 gene transfer against apoptosis induced by hypoxia in PC12 cells.

\section{Discussion}

Hypoxia-specific regulatory systems have been developed to regulate transgene expression in hypoxic tissues (3-8), in which gene expression is upregulated in a tissue-specific manner and reduced in normal tissues. Various copies of HRE have been developed to control therapeutic gene expression and all of these copies of HRE can upregulate the expression of downstream genes in response to hypoxia. Some studies have demonstrated that 5 copies of HRE derived from the human VEGF gene were optimal to mediate response to hypoxia $(5,16)$. However, other studies have found that 9 copies of the HRE from the human EPO gene were more effective than 3 or 6 copies (17). This discrepancy may be attributed to the different promoters used $(6,18)$. There are 2 main promoters used in hypoxia-specific regulatory systems, the minimal cytomegalovirus promoter (CMVmp) and SV40mp. Taking into account the results from our previous study (3), we selected 5 copies of the HRE from the human VEGF gene and SV40mp to construct a cassette to conditionally regulate NT-3 expression in response to hypoxia in this study. As expected, under the control of 5HRE, both the mRNA and protein expression of NT-3 significantly increased after hypoxia in PC12-5HRE-NT3 cells. An ELISA analysis revealed that the concentration of NT-3 protein in the conditioned medium of PC12-5HRE-NT3 cells in the hypoxic group increased by almost 10 -fold compared to the normoxic group. These findings indicate that the cassette composed of 5HRE and SV40mp is an available 'modulator' to regulate therapeutic gene expression in hypoxia-related diseases.

Different copies of HRE can decrease the basal promoter activity under normoxic conditions. As shown in a previous study, the 5HRE-3NRSE regulator cassette decreased the basal CMVmp activity by approximately $50-97 \%$ in comparison with that of the CMV construct in cultured cells under normoxic conditions (19). Moreover, almost no humanized Renilla GFP (hrGFP) expression occurred in the PC12 cells infected by Ad-5HRE-hrGFP under normoxic conditions (20). In vivo, Shen et al (4) found that the lacZ gene was not expressed in the normal areas of the AAVH9-lacZ-transduced mouse brain. Consistent with these data, we found that under normoxic conditions, the 5HRE-SV40mp construct had a significantly lower promoter activity compared with the SV40mp construct, as the basal SV40mp promoter activity was reduced by $92 \%$ from NT-3 mRNA expression, $83 \%$ from NT-3 protein expression and $91 \%$ from secreted NT-3 protein expression. Taken together, these data indicate that 5HRE is an ideal enhancer to perform hypoxia-specific gene expression. Therefore, 5HRE can effectively upregulate downstream gene expression under hypoxic conditions and only small or unexpected side-effects on the physiological process occur through suppression of the promoter under normal conditions.

The PC12 cell line has been extensively used as a model for the study of neurosecretion, neuronal signaling pathways, neuronal differentiation, neuroprotective effects of neurotrophin and ischemic tolerance (21). A number of studies have demonstrated that NT-3 exerts various effects on PC12 cells that have been subjected to various types of treatment, including hypoxia (21). Therefore, the PC12 cell line, which possesses neuron-like properties, is an ideal cell line to use to determine the effects of NT-3 and the bioactive functions of conditionally expressed NT-3.

Hypoxic injury in the PC12 cell model was induced with treatment at an oxygen concentration of $0.3 \%$ to mimic cerebral ischemic injury (22). Although HIF-1 $\alpha$ expression increased and peaked after hypoxia at $24 \mathrm{~h}$ in PC12 cells, the expression of downstream genes was induced in a time-dependent manner (20). Therefore, hypoxia for $48 \mathrm{~h}$ was used as a model of hypoxia-induced injury in PC12 cells, in which clear apoptotic responses could be induced and hypoxia-regulated NT-3 expression could be thoroughly induced to display the protective effects against apoptosis.

Caspase-3 plays a critical role in apoptotic execution (23) and hypoxia induces the cleavage of caspase- 3 through the p38/mitogen-activated protein kinase (MAPK)-dependent pathway (24). It has previously been reported that NT-3 can promote extracellular signal-regulated protein kinase/ MAPK and phosphatidylinositol 3-kinase/Akt phosphorylation, where NT-3 can exert its anti-apoptotic effects (25). In the present study, the decreased activation of p38 and caspase- 3 in the PC12-5HRE-NT3 cell group under hypoxic conditions indicated that both p38 and caspase-3 were involved in the reduction of apoptosis induced by hypoxia in PC12 cells, which was mediated by 5 HRE-NT3 gene transfer.

In conclusion, in this study, we successfully constructed a retroviral vector carrying 5HRE-NT3 and transferred it into PC12 cells (PC12-5HRE-NT3). Under hypoxic conditions, NT-3 expression was significantly upregulated by 5HRE in PC12-5HRE-NT3 cells. Conditionally expressed NT-3 reduced apoptosis induced by hypoxia in PC12 cells, which may be due to the depressed activation of p38 and caspase-3. These results suggest that the 5HRE-NT3 vector may be useful for the therapy of ischemic diseases, while avoiding side-effects.

\section{Acknowledgements}

This study was supported by the National Natural Science Foundation of China (Grant no. 81070998) and a fund from the State Key Laboratory of Medical Neurobiology, Shanghai (no. 10-03). The authors would like to thank Professors Julie Y.H. Chan and Samuel H.H. Chan from the Center for Translational Research in Biomedical Sciences, Chang Gung Memorial Hospital-Kaohsiung Medical Center, Taiwan for their invaluable comments and editorial support.

\section{References}

1. Beenken A and Mohammadi M: The FGF family: biology, pathophysiology and therapy. Nat Rev Drug Discov 8: 235-253, 2009. 
2. Lee RJ, Springer ML, Blanco-Bose WE, Shaw R, Ursell PC and Blau HM: VEGF gene delivery to myocardium: deleterious effects of unregulated expression. Circulation 102: 898-901, 2000.

3. Shi Q, Zhang P, Zhang J, et al: Adenovirus-mediated brainderived neurotrophic factor expression regulated by hypoxia response element protects brain from injury of transient middle cerebral artery occlusion in mice. Neurosci Lett 465: 220-225, 2009.

4. Shen F, Su H, Fan Y, et al: Adeno-associated viral-vectormediated hypoxia-inducible vascular endothelial growth factor gene expression attenuates ischemic brain injury after focal cerebral ischemia in mice. Stroke 37: 2601-2606, 2006.

5. Shibata T, Giaccia AJ and Brown JM: Development of a hypoxia-responsive vector for tumor-specific gene therapy. Gene Ther 7: 493-498, 2000.

6. Su H, Arakawa-Hoyt J and Kan YW: Adeno-associated viral vector-mediated hypoxia response element-regulated gene expression in mouse ischemic heart model. Proc Natl Acad Sci USA 99: 9480-9485, 2002.

7. Semenza GL: Targeting HIF-1 for cancer therapy. Nat Rev Cancer 3: 721-732, 2003.

8. Iyer NV, Kotch LE, Agani F, et al: Cellular and developmental control of $\mathrm{O}_{2}$ homeostasis by hypoxia-inducible factor 1 alpha. Genes Dev 12: 149-162, 1998.

9. Patapoutian A and Reichardt LF: Trk receptors: mediators of neurotrophin action. Curr Opin Neurobiol 11: 272-280, 2001.

10. Li X, Yang Z and Zhang A: The effect of neurotrophin-3/ chitosan carriers on the proliferation and differentiation of neural stem cells. Biomaterials 30: 4978-4985, 2009.

11. Lu H, Li M, Song T, et al: Retrovirus delivered neurotrophin-3 promotes survival, proliferation and neuronal differentiation of human fetal neural stem cells in vitro. Brain Res Bull 77: $158-164,2008$

12. Lu HX, Hao ZM, Jiao Q, et al: Neurotrophin-3 gene transduction of mouse neural stem cells promotes proliferation and neuronal differentiation in organotypic hippocampal slice cultures. Med Sci Monit 17: BR305-BR311, 2011.

13. Zhang ZH, Wang RZ, Li GL, et al: Transplantation of neural stem cells modified by human neurotrophin-3 promotes functional recovery after transient focal cerebral ischemia in rats. Neurosci Lett 444: 227-230, 2008.
14. Cristofaro B, Stone OA, Caporali A, et al: Neurotrophin-3 is a novel angiogenic factor capable of therapeutic neovascularization in a mouse model of limb ischemia. Arterioscler Thromb Vasc Biol 30: 1143-1150, 2010.

15. Shimazu K, Zhao M, Sakata K, et al: NT-3 facilitates hippocampal plasticity and learning and memory by regulating neurogenesis. Learn Mem 13: 307-315, 2006.

16. Shibata T, Giaccia AJ and Brown JM: Hypoxia-inducible regulation of a prodrug-activating enzyme for tumor-specific gene therapy. Neoplasia 4: 40-48, 2002.

17. Ruan H, Su H, Hu L, Lamborn KR, Kan YW and Deen DF: A hypoxia-regulated adeno-associated virus vector for cancerspecific gene therapy. Neoplasia 3: 255-263, 2001.

18. Mori N, Stein R, Sigmund O and Anderson DJ: A cell typepreferred silencer element that controls the neural-specific expression of the SCG10 gene. Neuron 4: 583-594, 1990.

19. Huang D, Desbois A and Hou ST: A novel adenoviral vector which mediates hypoxia-inducible gene expression selectively in neurons. Gene Ther 12: 1369-1376, 2005.

20. Hu HW, Li XK, Zheng RY, Xiao J, Zeng JQ and Hou ST: bFGF expression mediated by a hypoxia-regulated adenoviral vector protects PC12 cell death induced by serum deprivation. Biochem Biophys Res Commun 390: 115-120, 2009.

21. Hillion JA, Takahashi K, Maric D, Ruetzler C, Barker JL and Hallenbeck JM: Development of an ischemic tolerance model in a PC12 cell line. J Cereb Blood Flow Metab 25: 154-162, 2005.

22. Silver I and Erecinska M: Oxygen and ion concentrations in normoxic and hypoxic brain cells. Adv Exp Med Biol 454: 7-16, 1998.

23. Riedl SJ and Shi Y: Molecular mechanisms of caspase regulation during apoptosis. Nat Rev Mol Cell Biol 5: 897-907, 2004.

24. Morisco C, Marrone C, Trimarco V, et al: Insulin resistance affects the cytoprotective effect of insulin in cardiomyocytes through an impairment of MAPK phosphatase-1 expression. Cardiovasc Res 76: 453-464, 2007.

25. Liot G, Gabriel C, Cacquevel M, et al: Neurotrophin-3-induced PI-3 kinase/Akt signaling rescues cortical neurons from apoptosis. Exp Neurol 187: 38-46, 2004. 